\title{
Half-life of Ingested Antibodies in Anopheles Stephensi Mosquito Is Less Than 10 Hours
}

\section{Kazutoyo Miura ( $\nabla$ kmiura@niaid.nih.gov )}

National Institute of Allergy and Infectious Diseases https://orcid.org/0000-0003-4455-2432

\section{Deng Bingbing}

NIAID: National Institute of Allergy and Infectious Diseases

\section{Yonas T Gebremicale}

NIAID: National Institute of Allergy and Infectious Diseases

\section{Thao P Pham}

NIAID: National Institute of Allergy and Infectious Diseases

\section{Ababacar Diouf}

NIAID: National Institute of Allergy and Infectious Diseases

\section{Luwen Zhou}

NIAID: National Institute of Allergy and Infectious Diseases

\section{Emily Locke}

PATH Malaria Vaccine Initiative

\section{Randall S MacGill}

PATH Malaria Vaccine Initiative

\section{Carole A Long}

NIAID: National Institute of Allergy and Infectious Diseases

\section{Short report}

Keywords: antibody, longevity, half-life, Anopheles mosquito, malaria, transmission-blocking vaccine

Posted Date: October 5th, 2021

DOI: https://doi.org/10.21203/rs.3.rs-951322/v1

License: (c) (i) This work is licensed under a Creative Commons Attribution 4.0 International License. Read Full License 


\section{Abstract}

Background: A transmission-blocking vaccine (TBV) can be a useful tool to reduce malaria infection in an endemic area. For a TBV, elicited antibody (either by itself or working with complement) has a critical role in the mechanism of action, which for most known TBV targets, blockade will occur within the mosquito. However, no study has quantitively assessed the longevity of ingested antibody in Anopheles mosquito vectors.

Methods: A mixture of mouse or human monoclonal antibody (mAb), human red blood cells and human serum were fed to An. stephensi mosquitoes, and their midguts were collected at multiple time points ( 0 to 48 hours; 12 mosquitoes at each time point) after feeds. The reactivity of antibodies against target antigen (integrity of antigen-binding region of the antibody) in each midgut was assessed by ELISA. For one mouse mAb, integrity of antibody constant region was also determined by western blot (WB) with a mouse-specific secondary antibody.

Results: First, the half-life of mouse anti-Pfs $25 \mathrm{mAb}$, 4B7, was determined both by ELISA and WB in three independent assays. When the ELISA and WB signals were plotted against time after feed, both data reasonably fit one-phase exponential decay models $\left(R^{2} B \quad 0.70\right)$, and the half-lives were estimated as 8.6 hours by ELISA and 4.7 hours by WB. To determine whether the longevity was affected by target antigens or species of antibody, two human anti-Pfs25 mAbs (AB1245 and AB2544), one human anti-Pfs48/45 mAb (TB31F), and one mouse anti-Pfs230 mAb (15A4-1B12) were examined by ELISA in two or three independent assays. The ELISA results of each additional $\mathrm{mAb}$ also reasonably fit to a one-phase exponential decay model $\left(R^{2}\right.$ a 0.78$)$, and the half-lives of those mAbs were similar to that of $4 B 7$ (7.2 to 9.3 hours), except AB1245 which showed a half-life of 4.6 hours.

Conclusions: Depending on the methods of detection and mAbs used, the longevity of ingested antibody varied around 2 -fold, but all estimated half-lives were $<10$ hours. These data suggest a TBV with antibody dependent mechanism of action(s) is more likely to succeed when targeting earlier stages of parasites (or parasite interaction) in mosquitoes.

\section{Background}

Malaria is still a major health problem worldwide, and according to the World Malaria Report 2020, there were an estimated 229 million clinical cases, which resulted in 409,000 deaths in 2019 [1]. Transmissionblocking vaccines (TBVs) are projected to be a valuable tool to reduce malaria burden and may strongly accelerate malaria elimination efforts in low endemicity areas [2]. Generally, TBVs are designed to induce antibodies in a human host, and ingested antibodies will kill parasites or inhibit parasite growth in the mosquito host. Multiple phase 1 clinical trials have been conducted with Pfs25, Pvs25 and Pfs230-based vaccines [2], and the first phase 2 trial is underway in Mali (ClinicalTrials.gov Identifier: NCT03917654). Furthermore, a phase 1 trial with recombinant human anti-Pfs48/45 monoclonal antibody (mAb), TB31F, has been conducted in the Netherlands (NCT04238689). 
When a blood meal is taken by an Anopheles mosquito, excess water is removed, and the mosquito starts to secrete digestive enzymes within several hours [3]. The trypsin, aminopeptidase and a-glucosidase enzyme activities in An. stephensi peak at 24 to 30 hours after the blood meal (hour-post-feed, hpf), and total protein concentration of the whole midgut homogenate goes back to the baseline level around 60 hpf [4]. Therefore parasites must complete critical developmental steps such as gametogenesis (conversion from gametocytes to gametes), fertilization (to become zygotes), differentiation to and maturation of ookinetes, and then penetration of the midgut wall by the mature ookinetes within the $\sim 60$ hour window. In the case of Plasmodium falciparum, which is the most lethal Plasmodium species to infect humans, in general ookinete forms of parasites appear at $~ 10-20 \mathrm{hpf}$, peak at $\sim 24-36 \mathrm{hpf}$, then disappear by 50-60 hpf in the midguts, although the kinetics varies slightly depending on the mosquito species and temperature [5-8]. Therefore, if a mechanism of action of a TBV is to kill mature ookinetes or to prevent ookinete penetration, for example, the antibodies should maintain an effective concentration in the mosquito midguts for a minimum of 24-36 hours. On the other hand, if the mechanism of action is to prevent fertilization (fertilization usually happens before $\sim 2 \mathrm{hpf}[9]$ ), the antibody may not need to survive for a longer time in the mosquitoes' midguts. When An. stephensi mosquitoes were fed with test antibodies in their bloodmeals, the antibodies were detected at $24 \mathrm{hpf}$ by ELISA [10] or up to $48 \mathrm{hpf}$ by Immunofluorescence assay (IFA) [11]; thus, antibodies are considered to survive at effective concentrations for 24-36 hours in mosquito guts [12]. However, the former study assessed the level of antibody at a single time point (i.e., only at $24 \mathrm{hpf}$, no data at $0 \mathrm{hpf}$ ), and the latter study used a binomial readout (i.e., IFA positive or negative). Hence, quantitative conclusions should not be drawn from those preceding studies, i.e., what proportion of antibodies remains at a given time after feed. In this study, several mouse and human monoclonal antibody (mAb) samples were fed to An. stephensi mosquitoes, the midguts were collected at multiple time points after feeds ( 0 to $48 \mathrm{hpf}$ ), then the amount of antibody in each midgut was assessed by ELISA or western blot (WB). This study revealed that the half-life of ingested antibody in a mosquito midgut was $<10$ hours for all 5 different mAbs tested.

\section{Methods}

\section{Test materials}

The origin and characteristics of 4B7 (mouse anti-Pfs25 [13]), AB1245 (human anti-Pfs25 [14]), AB2544 (human anti-Pfs25 [15]), TB31F (human anti-Pfs48/45 [16]), and 15A4-1B12 (mouse anti-Pfs230 [17]) mAbs have been described elsewhere. The human serum and red blood cells used for the feeding experiments were purchased from Interstate Blood Bank (Memphis, TN, USA). The affinity-purified goat anti-mouse IgG $(H+L)$ specific antibody for WB was purchased from Sera Care (Cat \# 05-18-06, Milford, MA, USA). For ELISA, alkaline phosphatase labeled goat anti-human IgG (Cat \# 075-1006) and alkaline phosphatase labeled goat anti-mouse IgG (Cat \# 075-1806) antibodies were utilized (Kirkegaard \& Perry Lab, Gaithersburg, MD, USA).

\section{Mosquito feeding experiments}


One hundred $\mathrm{mL}$ of uninfected human packed red blood cells (RBCs) were mixed with $100 \mathrm{~mL}$ of nonheat inactivated human serum and $60 \mathrm{~mL}$ of test $\mathrm{mAb}$ at $1 \mathrm{mg} / \mathrm{ml}$ in $1 \mathrm{x}$ phosphate buffered saline (1xPBS). The $\mathrm{mAb}$ concentration in the final mixture was $375 \mathrm{mg} / \mathrm{mL}$ in the liquid volume. The mixture was immediately fed to $\sim 60$ female An. stephensi mosquitoes (4-days-old) through a membrane-feeding apparatus, as performed for a standard membrane feeding assay (SMFA) described previously [18]. The mosquitoes were dissected ( $\mathrm{n}=12$ per time per assay) at indicated timepoints (from 0 to $48 \mathrm{hpf}$ ), and each midgut was collected in a tube with $20 \mathrm{~mL}$ of 1 xPBS, snap frozen with dry ice, and kept at $-80^{\circ} \mathrm{C}$ until used. The midguts without blood at the time of dissection were not collected. For each mAb, two or three independent experiments were performed, except 4B7, which were tested a total of 4 independent assays. For maintenance, mosquitoes were given 10\% glucose solution ad libitum, except for the 15-18 hours of starvation before the feeds, and maintained in $75 \%$ humidity at $27^{\circ} \mathrm{C}$ throughout the experiments.

\section{ELISA}

The basic methodology of regular ELISA has been published elsewhere [19]. For anti-Pfs25 mAbs (4B7, $A B 1245$ and AB2544), the ELISA plates were coated with Pfs25MF1E (a full-length Pfs25 recombinant protein [20]). For TB31F, R06C (6C-fragment of Pfs48/45 fused with GLURP antigen [21]) was used as a coating antigen, and Pfs230C1 (N-terminal of Pfs230 [17]) for 15A4-1B12 mAb. On the day of ELISA, for each $\mathrm{mAb}$ in each experiment, all midgut samples collected at multiple time points were thawed at the same time, homogenized by vortex, and diluted at mAb-specific dilutions (1:2,000 to 1:8,000 dilutions). The dilution factors were determined by preliminary experiments so the majority of $0 \mathrm{hpf}$ samples showed ODs of 1.5-2 in the standardized assay at the optimized dilution. The thaw, homogenization and dilution steps were conducted within 1 hour, and ELISA was performed immediately after the sample preparation.

\section{SDS-PAGE and Western blotting (WB)}

The basic methodologies for SDS-PAGE and WB have been described elsewhere [22], and the assays were conducted only for 4B7 mAb samples. In brief, just before SDS-PAGE, all midgut samples from a single experiment were thawed at the same time, homogenized by vortex, mixed with LDS sample buffer (1:32.5 dilution), and loaded to a 4-12\% Bis-Tris SDS-PAGE gel under non-reducing conditions. The dilution factor was determined by preliminary experiments, where all $0 \mathrm{hpf}$ samples showed less than top saturation density in the WB at the 1:32.5 dilution. In each gel, 4 samples collected at each of all timepoints from a single experiment were loaded (e.g., 4 samples each at 0, 3, 20 and $27 \mathrm{hpf}$, a total of 16 samples were loaded into a single gel), and 3 to 4 gels were utilized per experiment. Following the SDS-PAGE, 4B7 mAb protein was transferred onto nitrocellulose membranes and was detected by the affinity-purified goat antimouse IgG $(\mathrm{H}+\mathrm{L})$ specific antibody (1:2,000 dilution). The band density of each sample was quantified using Amersham Imager 680 (GE Healthcare, Chicago, IL, USA). Serial dilutions of known concentrations of $4 B 7 \mathrm{mAb}$ in RBCs and human serum mixtures were processed in the same way to generate a standard 
curve, and a density value of test sample was converted to $4 \mathrm{~B} 7 \mathrm{mAb}$ concentrations using the standard curve.

\section{Statistical analysis}

For the WB results, in each blot, an arithmetic mean of $4 B 7 \mathrm{mAb}$ concentration of $0 \mathrm{hpf}$ samples (4 samples in each blot) was calculated, and a relative $4 B 7 \mathrm{mAb}$ concentration of test sample from the other timepoints in the same blot was determined as percent (\%) relative to the average $0 \mathrm{hpf}$ value (\%Ohpf). Similar to the WB analysis, ELISA result of each non-0-hpf sample was converted to \%Ohpf by calculating the relative amount against the average $0 \mathrm{hpf}$ samples in the same experiment $(12$ samples in each experiment). If the antibody concentrations were too low to determine (i.e, less than the minimal detection limit of assay), \%0hpf value was assigned as zero, and the lowest positive values were 0.04 \%0hpf for WB and $1.9 \% 0 \mathrm{hpf}$ for ELISA. To determine the best-estimate of half-life (and the $95 \%$ confidence interval, $95 \% \mathrm{Cl}$ ), the \% $0 \mathrm{hpf}$ values were plotted against their hpf values individually, and a one-phase exponential decay model was utilized to determine the best-fit curve. To compare half-life of 4B7 between ELISA and WB, a F-test was used. An F-test was utilized to compare half-lives of two mAbs (only for ELISA data) at a time, then Bonferroni corrected $p$-values were calculated to adjust for multiple comparisons. All statistical tests were performed in Prism 8 (GraphPad Software), and $p$-values $<0.05$ were considered significant.

\section{Results}

To determine a half-life of ingested antibody, mouse anti-Pfs $25 \mathrm{mAb} 4 \mathrm{~B} 7$ was mixed with human uninfected RBC and human serum, and fed to An. stephensi mosquitoes. The midguts from blood-fed mosquitoes were collected ( $n=12$ at each time point) at 0,24 and 41 hour-post-feed (hpf), then amount of 4B7 mAb in each midgut was assessed by ELISA and WB (Exp 1 in Fig. 1). There were large mosquito-tomosquito variations at $0 \mathrm{hpf}$; a maximum of $\sim 3$-fold difference measured by ELISA (58 to $150 \% 0 \mathrm{hpf}$ ) and 2 -fold difference by WB (75 to $129 \% 0 \mathrm{hpf}$ ). At $41 \mathrm{hpf}$, all samples showed less than minimum detection level of signals (i.e., \%0hpf was zero) in both assays. Therefore, two more independent experiments (Exp 2 and 3) were performed at 0, 3, 20 and $27 \mathrm{hpf}$ (all original \%0hpf data are shown in Table S1). When individual midgut data from all three experiments were combined, the correlation between \%0hpf and hpf was reasonably explained by a one-phase exponential decay model; $R^{2}=0.82$ for ELISA, and $\mathrm{R}^{2}=0.70$ for WB. The estimated half-life of $4 \mathrm{~B} 7 \mathrm{mAb}$ by ELISA was 8.6 hours $(95 \% \mathrm{Cl} ; 7.4$ to 10.0 hours), and 4.7 hours $(95 \% \mathrm{Cl}, 3.4$ to 6.4 hours $)$ by WB. There was a significant difference $(p=0.0003)$ between the two methods.

Next, we investigated whether the longevity was affected by target antigens or species of antibody. Two human anti-Pfs25 mAbs (AB1245 and AB2544), one human anti-Pfs48/45 mAb (TB31F), and one mouse anti-Pfs230 mAb (15A4-1B12) were examined. All human mAbs are recombinant mAbs with IgG1 backbone [14-16], while 4B7 and 15A4-1B12 mAbs were produced from mouse hybridoma cells [13, 17]. For each mAb, two independent assays were conducted; the first experiment at 0-17-24-48 hpf, and then 
the second one at 0-5-23-28 hpf (Fig. 2: all original \%0hpf values are seen in Table S2). Since WB method cannot distinguish the recombinant human mAbs and human antibodies in the human serum, only the ELISA method was utilized for the comparison. Similar to $4 \mathrm{~B} 7 \mathrm{mAb}$, none of the mAbs were detected at $48 \mathrm{hpf}$, and the data also fit one-phase exponential decay models; $\mathrm{R}^{2}=0.92,0.78,0.90$ and 0.94 for $A B 1245, A B 2544, T B 31 F$ and 15A4-1B12, respectively. While the half-lives of $A B 2544$ (9.3 hours; $95 \% \mathrm{Cl}$, 7.8 to 11.0$)$, TB31F (7.2 hours; $95 \% \mathrm{Cl}, 6.5$ to 7.9$)$ and $15 \mathrm{~A} 4-1 \mathrm{~B} 12$ (8.7 hours; $95 \% \mathrm{Cl}, 7.7$ to 9.7$)$ mAbs were not different from the half-life of $4 B 7$ ( $p>0.129)$, the half-life of $A B 1245$ mAb was shorter (4.3 hours; $95 \% \mathrm{Cl}, 3.6$ to $5.0 ; p<0.0001$ compared to $4 \mathrm{~B} 7)$. To confirm the shorter half-life of $A B 1245$ mAb was not due to assay variability, the mAb was tested one more time at 0-5-23-28 hpf. Considering the large mosquito-to-mosquito variation within an experiment, the two independent experiments provided indistinguishable results; average \% $0 \mathrm{hpf}$ of the second experiment were 45, 0, and 0 at 5,23 and $28 \mathrm{hpf}$, respectively, while 53, 1 and 0 in the third experiment. The half-life of $A B 1245 \mathrm{mAb}$ from three experiments was estimated as 4.6 hours $(95 \% \mathrm{Cl}, 4.0$ to $5.3 ; \mathrm{p}<0.0001$ compared to $4 \mathrm{~B} 7)$. When half-lives of all $5 \mathrm{mAbs}$ were compared to each other, only AB1245 mAb showed a significantly shorter half-life when compared to the other $4 \mathrm{mAbs}(\mathrm{p}<0.0001 \mathrm{for}$ all), and there was no difference among the remaining 4 mAbs $(p>0.064)$.

\section{Discussion}

To the best of our knowledge, this is the first study which has quantitively evaluated the longevity of ingested antibody in Anopheles mosquitoes. This study reveals a half-life of ingested antibody is $<10$ hours, regardless of method of detection, antibody species and TBV antigen.

To determine the half-life of ingested antibodies, we utilized two different methods (ELISA and WB) to test the longevity of a mouse mAb (4B7), and 5 different mAbs were compared by ELISA. When 4B7 mAbs were evaluated by the two methods, there was a significant difference in their half-lives. The two methods assess the integrity of different parts of antibodies (variable region of antibody by ELISA, and constant region by WB), and the two different parts in the mAb could have different stability against the harsh condition in the mosquitoes' midguts. Likewise, half-lives determined by ELISA were not necessarily the same for all mAbs. The different mAbs have different amino acids sequences in their variable regions, thus variable rates of $m A b$ decay are not surprising. In this study, the proportion of RBC, serum and $m A b$ in the feeding mixture was set as we routinely perform SMFA, and the concentration of mAbs given and assay conditions were optimized so that quantitative change of test antibody in midguts could be detected by the ELISA and WB. While no antibody signal was detected at $48 \mathrm{hpf}$ in this study, these data do not mean all ingested antibodies would be digested or degraded within 48 hours. Antibody signal may be detected at $48 \mathrm{hpf}$ if larger amounts of antibodies are given to the mosquitoes, and/or an assay with higher sensitivity is utilized. The fundamental difference of this study from the preceding two studies [10, 11 ] is to determine the half-life. If a half-life of antibody is 10 hours, and the amount of antibody measured by ELISA (or WB) in a midgut linearly correlates with the functional activity, $>95 \%$ of ingested antibodies would lose activity within 48 hours. Since one of the biggest challenges in vaccine 
development is to induce (and maintain) high antibody titers in vaccinated humans, the results of this study could have a considerable impact on the future of TBV antigen discovery and selection. For example, if a TBV can induce high titers in immunized animals or humans, regardless of timing of the target antigen expression, the TBV could show a promising impact on parasite transmission. On the other hand, in the situation where a TBV can induce only lower titers, due to immunized species and/or the nature of the target antigen, the impact of antibody degradation within the midguts on transmissionblocking activity could be an important factor to consider.

There are several limitations in this study. First, the study utilized only An. stephensi, therefore, the half-life of antibody in other mosquito species was not evaluated. When the same human serum (including pre-fertilization polyclonal antibodies), anti-Pfs48/45 (pre-fertilization), anti-Pfs230 (prefertilization) and anti-Pfs25 (post-fertilization) monoclonal antibodies were tested by SMFA using both An. stephensi and An. gambiae mosquitoes, there was virtually no difference in \% inhibition in oocyst density for all pre-fertilization antibodies [23]. On the other hand, anti-Pfs 25 mAb 4B7 showed higher inhibitions in An. stephensithan in An. gambiae. The same mosquito species effect for 4B7 mAb was also observed in SMFA conducted in another laboratory [23]. The results suggest the half-life of antibodies in An. gambiae might be even shorter than that in An. stephensi (therefore, weaker inhibitions were observed in An. gambiae). Second, the assay was done without parasites, because a portion of test antibodies may bind with target antigens expressed by parasites in mosquitoes' midguts, and it could complicate the interpretation of results. Further study is required to answer whether the existence of parasites in the midguts has an effect on the half-life of ingested TBV antibodies.

\section{Conclusion}

In this study 5 different human or mouse mAbs were fed to An. stephensi mosquitoes to determine their half-life in mosquitoes. The half-lives of all mAbs were estimated as less than 10 hours. These results suggest a TBV which targets an earlier stage of parasite antigen(s) (or parasite-mosquito interaction) in mosquitoes has a higher chance of success, unless antibodies against a later stage of parasite antigen requires a much lower quantity to show functional activity. This aspect should be considered in future TBV candidate discovery and antigen selection.

\section{Abbreviations}

hpf, hour-post-feed; mAb, monoclonal antibody; RBC, red blood cell; SMFA, standard membrane feeding assay; TBV, transmission-blocking vaccine; WB, western blot; \%0hpf, percent relative to the average $0 \mathrm{hpf}$ value.

\section{Declarations}

Ethics approval and consent to participate 
Not applicable

\section{Consent for publication}

Not applicable

\section{Availability of data and materials}

All data analyzed during this study are included in this published article and its supplementary information files.

\section{Competing interests}

The authors declare that they have no competing interests.

\section{Funding}

This study was supported by the Intramural Research Program of the National Institute of Allergy and Infectious Diseases, NIH and PATH's Malaria Vaccine Initiative.

\section{Author contributions}

KM: conception and design, analysis and interpretation of data, and draft the paper. BD, YTG, TPP, AD and LZ: acquisition of data, and revision of article. EL and RSM: experimental design, analysis and interpretation of data, and revision of article. CAL: conception and design, interpretation of data, revision of article. All authors read and approved the final manuscript.

\section{Acknowledgements}

The graphical abstract was created with BioRender.com.

\section{References}

1. World Health Organization. World malaria report 2020. https://wwwwhoint/publications/i/item/9789240015791.

2. Miura K, Tachibana M, Takashima E, Morita M, Kanoi BN, Nagaoka H, et al. Malaria transmissionblocking vaccines: wheat germ cell-free technology can accelerate vaccine development. Expert Rev Vaccines. 2019;18:1017-27

3. Vaughan JA. Population dynamics of Plasmodium sporogony. Trends Parasitol. 2007;23:63-70

4. Billingsley PF, Hecker H. Blood digestion in the mosquito, Anopheles stephensi Liston (Diptera: Culicidae): activity and distribution of trypsin, aminopeptidase, and alpha-glucosidase in the midgut. J Med Entomol. 1991;28:865-71 
5. Vaughan JA, Noden BH, Beier JC. Population dynamics of Plasmodium falciparum sporogony in laboratory-infected Anopheles gambiae. J Parasitol. 1992;78:716-24

6. Noden $\mathrm{BH}, \mathrm{Kent} \mathrm{MD}$, Beier JC. The impact of variations in temperature on early Plasmodium falciparum development in Anopheles stephensi. Parasitology. 1995;111 ( Pt 5):539-45

7. Robert V, le Goff G, Gouagna LC, Sinden M, Kieboom J, Kroneman R, et al. Kinetics and efficiency of Plasmodium falciparum development in the midguts of Anopheles gambiae, An. funestus and An. nili. Ann Trop Med Parasitol. 1998;92:115-8

8. Chege GM, Beier JC. Immunodetection of Plasmodium falciparum zygotes and ookinetes in Anopheles blood meals. J Am Mosq Control Assoc. 1994;10:419-22

9. Bennink S, Kiesow MJ, Pradel G. The development of malaria parasites in the mosquito midgut. Cell Microbiol. 2016;18:905-18

10. Lackie AM, Gavin S. Uptake and persistence of ingested antibody in the mosquito Anopheles stephensi. Med Vet Entomol. 1989;3:225-30

11. Vaughan JA, Do Rosario V, Leland P, Adjepong A, Light J, Woollett GR, et al. Plasmodium falciparum: ingested anti-sporozoite antibodies affect sporogony in Anopheles stephensi mosquitoes. Experimental parasitology. 1988;66:171-82

12. Sinden RE. Targeting the parasite to suppress malaria transmission. Adv Parasitol. 2017;97:147-85

13. Barr PJ, Green KM, Gibson HL, Bathurst IC, Quakyi IA, Kaslow DC. Recombinant Pfs25 protein of Plasmodium falciparum elicits malaria transmission-blocking immunity in experimental animals. $J$ Exp Med. 1991;174:1203-8

14. Scally SW, McLeod B, Bosch A, Miura K, Liang Q, Carroll S, et al. Molecular definition of multiple sites of antibody inhibition of malaria transmission-blocking vaccine antigen Pfs25. Nat Commun. 2017;8:1568

15. McLeod B, Miura K, Scally SW, Bosch A, Nguyen N, Shin H, et al. Potent antibody lineage against malaria transmission elicited by human vaccination with Pfs25. Nat Commun. 2019;10:4328

16. Kundu P, Semesi A, Jore MM, Morin MJ, Price VL, Liang A, et al. Structural delineation of potent transmission-blocking epitope I on malaria antigen Pfs48/45. Nat Commun. 2018;9:4458

17. Lee SM, Plieskatt J, Krishnan S, Raina M, Harishchandra R, King CR. Expression and purification optimization of an N-terminal Pfs230 transmission-blocking vaccine candidate. Protein Expr Purif. 2019;160:56-65

18. Miura K, Deng B, Tullo G, Diouf A, Moretz SE, Locke E, et al. Qualification of Standard MembraneFeeding Assay with Plasmodium falciparum Malaria and Potential Improvements for Future Assays. PloS one. 2013;8:e57909

19. Miura K, Orcutt AC, Muratova OV, Miller LH, Saul A, Long CA. Development and characterization of a standardized ELISA including a reference serum on each plate to detect antibodies induced by experimental malaria vaccines. Vaccine. 2008;26:193-200 
20. Farrance CE, Chichester JA, Musiychuk K, Shamloul M, Rhee A, Manceva SD, et al. Antibodies to plant-produced Plasmodium falciparum sexual stage protein Pfs25 exhibit transmission blocking activity. Hum Vaccin. 2011;7:191-8

21. Singh SK, Roeffen W, Andersen G, Bousema T, Christiansen M, Sauerwein R, et al. A Plasmodium falciparum 48/45 single epitope R0.6C subunit protein elicits high levels of transmission blocking antibodies. Vaccine. 2015;33:1981-6

22. Lee SM, Wu CK, Plieskatt J, McAdams DH, Miura K, Ockenhouse C, et al. Assessment of Pfs 25 expressed from multiple soluble expression platforms for use as transmission-blocking vaccine candidates. Malar J. 2016;15:405

23. Eldering M, Bompard A, Miura K, Stone W, Morlais I, Cohuet A, et al. Comparative assessment of An. gambiae and An. stephensi mosquitoes to determine transmission-reducing activity of antibodies against P. falciparum sexual stage antigens. Parasit Vectors. 2017;10:489

\section{Figures}
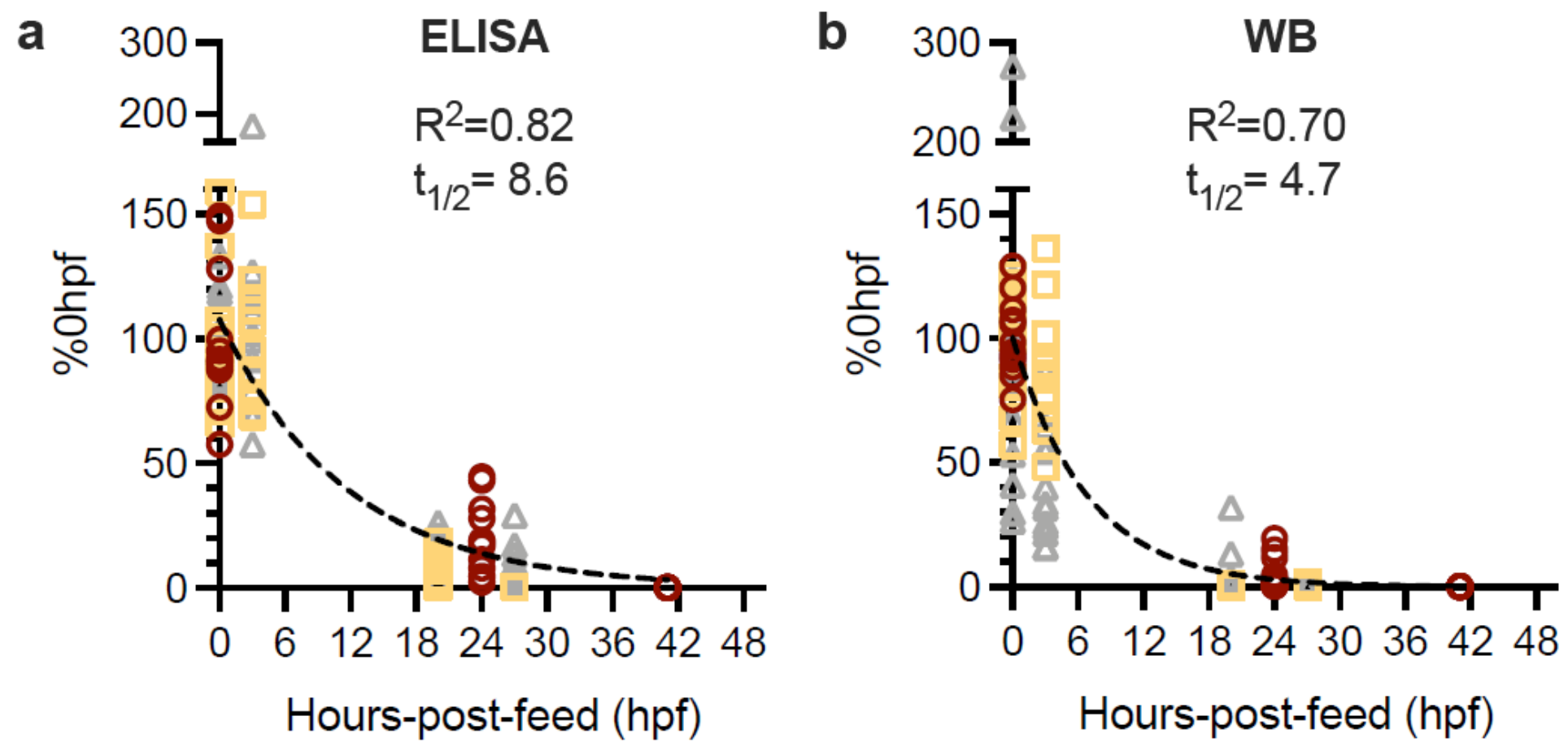
○ $\operatorname{Exp} 1$
$\operatorname{Exp} 2$
$\operatorname{Exp} 3$

Figure 1

Half-life of ingested $4 B 7$ mAb was $<10$ hours. Mixtures of $4 B 7 m A b$, human RBC and human serum were fed to Anopheles stephensi mosquitoes, and their midguts were collected at indicated time points (hourpost-feed, hpf). 4B7 mAb concentration in each midgut was determined by ELISA (a) or Western blot (WB, b), then the relative amount against the average of $0 \mathrm{hpf}$ samples (\%0hpf) was calculated. The midguts 
were harvested at 0-24-41 hpf in the first experiment (Exp 1), and at 0-3-20-27 hpf in the second and third experiments (Exp 2 and 3). Individual midgut data (dots), best-fit curve (dotted line), goodness of fit (R2) and estimated half-life (t1/2) are shown.

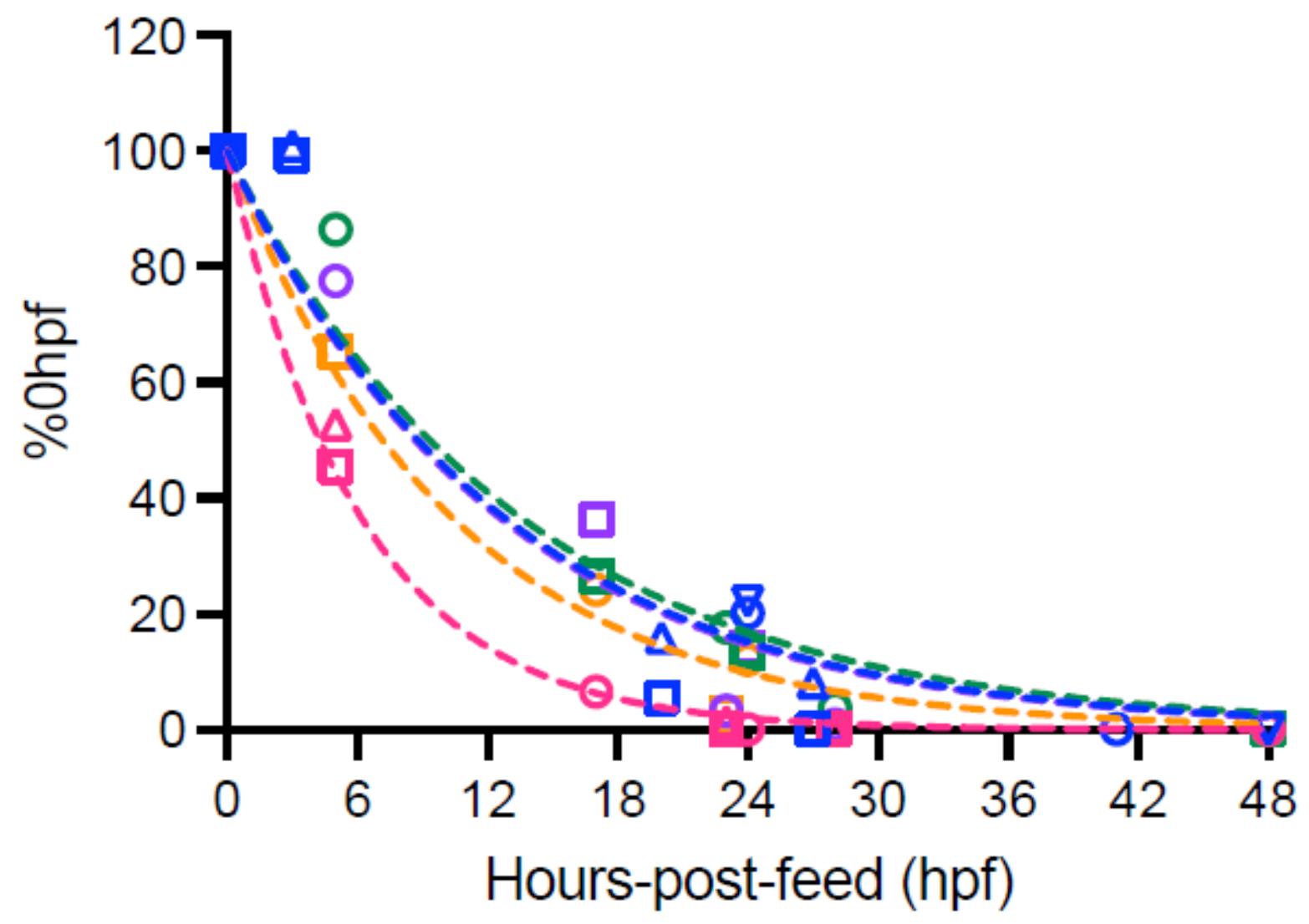

$$
\begin{aligned}
& \text {--• 4B7 --• AB1245 --. AB2544 } \\
& \text {-- TB31F -.• 15A4-1B12 }
\end{aligned}
$$

Figure 2

Half-lives of ingested 4 more mAbs were similar or shorter than half-life of 4B7. Mixtures of indicated mAb (AB1245, AB2544, TB31F or 15A4-1B12), human RBC and human serum were fed to An. stephensi mosquitoes, and their midguts were collected at 0-17-24-48 hpf in the first experiment, and at 0-5-23-28 $\mathrm{hpf}$ in the second experiment. For AB1245 mAb, the third experiment was performed at 0-5-23-28 hpf again. For 4B7 mAb, in addition to the three experiments shown in Fig.1, the fourth experiment was conducted at 0-24-48 hpf. For each mAb, the average of \%Ohpf values at each time point in each experiment (dots) are shown with the best fit-curve (dotted-line) calculated using \%0hpf values of individual midguts. 


\section{Supplementary Files}

This is a list of supplementary files associated with this preprint. Click to download.

- Graphicalimage.png

- TableS1.xIsx

- Tables2.xlsx 